University of New Hampshire

University of New Hampshire Scholars' Repository

Physics Scholarship

Physics

$12-1-2007$

\title{
Separation of spatial and temporal structure of auroral particle precipitation
}

\author{
A. Boudouridis \\ Harlan E. Spence \\ Boston University, harlan.spence@unh.edu
}

Follow this and additional works at: https://scholars.unh.edu/physics_facpub

Part of the Physics Commons

\section{Recommended Citation}

Boudouridis, A., and H. E. Spence (2007), Separation of spatial and temporal structure of auroral particle precipitation, J. Geophys. Res., 112, A12217, doi:10.1029/2007JA012591.

This Article is brought to you for free and open access by the Physics at University of New Hampshire Scholars' Repository. It has been accepted for inclusion in Physics Scholarship by an authorized administrator of University of New Hampshire Scholars' Repository. For more information, please contact Scholarly.Communication@unh.edu. 


\title{
Separation of spatial and temporal structure of auroral particle precipitation
}

\author{
A. Boudouridis ${ }^{1}$ and H. E. Spence ${ }^{2}$ \\ Received 12 June 2007; revised 1 September 2007; accepted 26 September 2007; published 22 December 2007.
}

[1] Knowledge of the dominant temporal and spatial scales of auroral features is instrumental in understanding the various mechanisms responsible for auroral particle precipitation. Single spacecraft data always suffer from temporal/spatial ambiguity. In an effort to separate the temporal and spatial variations of the aurora, we use electron and ion precipitation data from two co-orbiting satellites, F6 and F8 of the Defense Meteorological Satellite Program (DMSP). The two spacecraft have almost identical polar orbits with a small difference in period. As a result the time difference between the two measurements varies with time. We use two statistical tools in order to determine the most probable lifetimes and spatial dimensions of the prevalent auroral features. The first tool is cross-correlation analysis between the magnetic latitude series of electron and ion, number and energy fluxes measured by the two DMSP spacecraft. As one spacecraft overtakes the other, the variable time lag between the two measurements results in different cross-correlation of the two series. We explore the dependence of this variation on the time lag between the satellites. We find that the electron precipitation exhibits a decreasing correlation between the two spacecraft with increasing time lag, whereas there is only a small similar effect for the ion precipitation data. The second statistical tool is cross-spectral analysis, for which we compute the so-called coherence function as a function of frequency (or inverse wavelength) and hence size of the auroral features. The coherence function is a measure of the stability of auroral features of different sizes. We investigate its variation as a function of the time separation between the two measurements. We show that the coherence function of both electrons and ions remains high for up to 1.5 min spacecraft separations for all features larger than about $100 \mathrm{~km}$ in width. For smaller features the coherence is lower even for time lags of a few seconds. The results are discussed in the context of characteristic temporal and spatial auroral scales deduced from complementary studies and expected from theory.

Citation: Boudouridis, A., and H. E. Spence (2007), Separation of spatial and temporal structure of auroral particle precipitation, J. Geophys. Res., 112, A12217, doi:10.1029/2007JA012591.

\section{Introduction}

[2] The identification and evaluation of characteristic sizes and size distributions of discrete auroral features [Chiu and Schulz, 1978; Chiu and Cornwall, 1980; Lyons, 1980, 1981; Chiu et al., 1981; Chiu, 1986; Lotko et al., 1987] were important theoretical steps toward our understanding of the physical processes responsible for auroral phenomena. It is widely accepted [e.g., Lyons, 1992; Newell et al., 1996, and references therein] that discrete auroral features, such as auroral arcs, are the result of particle acceleration by quasistatic, field-aligned potential drops. It is also broadly acknowledged that a characteristic spatial scale of $50-$

\footnotetext{
${ }^{1}$ Department of Atmospheric and Oceanic Sciences, University of California, Los Angeles, California, USA.

${ }^{2}$ Center for Space Physics, Boston University, Boston, Massachusetts, USA.

Copyright 2007 by the American Geophysical Union. 0148-0227/07/2007JA012591
}

$100 \mathrm{~km}$ (at ionospheric altitudes) results naturally from a resistive mapping of the magnetospheric convection electric field into the ionosphere [Chiu and Cornwall, 1980; Lyons, 1980; Weimer et al., 1985; Lotko et al., 1987].

[3] Analyses of the spatial spectral distributions of electric and magnetic field variations in the ionosphere [Weimer et al., 1985, 1987; Reiff et al., 1988; Basu et al., 1988] have revealed the presence of structure over a range of spatial scale, including spatial features far smaller than the theoretical resistive scale length. A statistical study of $1.5 \times 10^{8}$ individual electron DMSP spectrograms by Newell et al. [1996] reveals an exponential distribution of precipitation scale sizes, from the DMSP resolution of $\sim 7 \mathrm{~km}$ up to about $100 \mathrm{~km}$, with an average latitudinal width of $28-$ $35 \mathrm{~km}$. Knudsen et al. [2001] conducted a statistical study of the width of 3126 mesoscale optical auroral arcs using an all-sky camera. They found a wide range of scale sizes $(1.7-40 \mathrm{~km})$ with a distribution peak at $18 \pm 9 \mathrm{~km}$, and a sharp cutoff in occurrence rate below $8 \mathrm{~km}$. Spatial scales even smaller than this have been observed, down to $100 \mathrm{~m}$ 
[Maggs and Davis, 1968], although high resolution particle data from the Freja satellite indicate that features smaller than $\sim 1 \mathrm{~km}$ are rare [Boehm et al., 1994]. Large statistical studies of intense electric fields using low-altitude Freja data [Karlsson and Marklund, 1996] and high-altitude Cluster data [Johansson et al., 2005] have shown that the most common spatial scales of electric field features are 1$5 \mathrm{~km}$ and 4-5 km, respectively, although their size distributions extend up to $10-20 \mathrm{~km}$.

[4] The early observational results stimulated theorists to consider processes which led to a spectrum of auroral spatial scales [Chiu, 1986, 1987; Lotko et al., 1987; Gorney, 1989] and to reexamine the temporal stability of the proposed auroral processes [e.g., Cornwall, 1990]. An excellent review of the early work on theoretical predictions of auroral arc thicknesses is presented by Borovsky [1993]. Further reviews on magnetosphere-ionosphere coupling and auroral arc formation can be found in the work of Lysak [1990] and Lyons [1992], respectively.

[5] It is well known [Knight, 1973; Lyons, 1980] that a field-aligned (parallel) current in the upward current region is linearly related to the parallel potential drop (the downward current region is known to be very dynamic and complex [Vedin and Rönnmark, 2005, and references therein]), that is

$$
J_{\|}=Q\left(\phi-\phi_{e}\right)
$$

where $\phi_{e}$ is the equatorial electrostatic potential, $\phi$ is the ionospheric electrostatic potential, and $Q$ is the currentpotential parameter $\left(Q \sim 0.1-0.2 \mathrm{~cm}^{-1} \mathrm{~s}^{-1}\right)$. The current conservation equation for this current [e.g., Lyons, 1980; Cornwall, 1988],

$$
\nabla \cdot\left(\sum_{p} \nabla \phi\right)=Q\left(\phi-\phi_{e}\right)
$$

leads to the definition of the ionospheric resistive scale length [Chiu and Cornwall, 1980; Lyons, 1980]

$$
\Lambda=\left(\frac{\Sigma_{p}}{Q}\right)^{1 / 2}
$$

where $\Sigma_{p}$ is the height-integrated Pedersen conductivity. This scale length is often thought of as a characteristic latitudinal scale length for auroral features because equatorial electric fields with gradient scale lengths shorter than $\Lambda$ cannot map perfectly into the ionosphere [Weimer et al., 1985; Lotko et al., 1987]. For typical ionospheric conditions, this length is on the order of $50-100 \mathrm{~km}$. It has been identified observationally through power spectral analysis of auroral electric fields [Weimer et al., 1985, 1987], power spectral analysis of precipitating electron flux [Gorney, 1989], and in comparison of high- and low-altitude electric field data [Reiff et al., 1988].

[6] Cornwall [1990] investigated the temporal stability of auroral arcs and identified several physically relevant timescales, each of which relates to a physical mechanism leading to a departure from equilibrium. These are as follows:

[7] 1. The first timescale is the ionization timescale

$$
T_{I}=\frac{e N}{\Gamma Q \Delta \phi} \simeq 30 \mathrm{~s}
$$

where $N$ is the height-integrated ionospheric plasma density $\left(N \sim 10^{12} \mathrm{~cm}^{-2}\right), \Gamma$ is the average number of electron-ion pairs produced by an incident auroral primary electron $(\Gamma \sim$ $30)$, and $\Delta \phi$ is the characteristic parallel electrostatic potential drop $(\Delta \phi \sim 1.5 \mathrm{kV})$.

[8] 2. The second timescale is the dissociative recombination timescale

$$
T_{D R}=(2 \alpha N)^{-1} \simeq 50 \mathrm{~s}
$$

where $\alpha$ is the height-integrated dissociative recombination rate $\left(\alpha \sim 10^{-14} \mathrm{~cm}^{2} \mathrm{~s}^{-1}\right)$.

[9] 3. The third timescale is the $\mathbf{E} \times \mathbf{B}$ drift transport timescale

$$
T_{E \times B}=\frac{B \Lambda^{2}}{c \Delta \phi} \simeq 50 \mathrm{~s}
$$

where $B$ is the ionospheric magnetic field magnitude. The characteristic time for this equation was computed assuming that the auroral arc scale size was the resistive scale size. In the above equations, $c$ and $e$ are the speed of light and the electronic charge, respectively.

[10] These temporal and spatial scales can vary since many of the above mentioned parameters can have a considerable variation from their usual values depending on the conditions in the high-latitude ionosphere. The smallest of the three timescales will be the one to dominate. Cornwall [1990] suggested that one reason for such a variation can be a low-energy aurora that cannot reach altitudes where dissociative recombination is important, and thus $\alpha$ is smaller than usual yielding a long dissociative recombination timescale $T_{D R}$. He goes on to argue that instability would occur in auroral features when and where the ionization rate exceeds the dissociative recombination rate, particularly if $\mathbf{E} \times \mathbf{B}$ drift transport is ineffective in stabilizing the increase in ionization which accompanies the auroral precipitation. Each of the physically significant timescales outlined by Cornwall [1990] is on the order of a few tens of seconds. Even for a given mechanism, the precise timescale will vary from feature to feature as noted above, depending on the fundamental characteristics of a particular auroral arc, both in space and time. Nevertheless, owing to gross similarity of timescales for each process, time variability alone cannot be used to identify uniquely a destabilization mechanism without additional information.

[11] In situ observations of both the spatial and temporal variability of auroral particle precipitation are exceptionally rare, as simultaneous measurements of the same features from at least two adjacent platforms are required. Single low-altitude satellites cannot separate temporal from spatial variation, and even the best conjunctions of two lowaltitude spacecraft are typically separated too widely in time and/or space to be of much help in exploring the temporal and spatial scales discussed above. An inherent uncertainty is imposed observationally when measuring and inferring arc characteristics from a single spacecraft which rapidly traverses the ionosphere. An observed feature in a time series suffers the ambiguity between a strictly spatial structure that is sampled along the satellite trajectory or a temporally evolving structure. In reality, both effects are 
probably at work. In the end, a single spacecraft yields data whose interpretation can put reasonable bounds on the extent to which features are temporal or spatial but cannot do so definitively. In order to better separate these effects, one can employ faster duty cycle instruments as is done on the Fast Auroral Snapshot (FAST) small explorer mission [e.g., Ergun et al., 1998, 2002, 2004]. While FAST is revolutionary in its ability to probe auroral phenomena, it cannot avoid the limitations inherent to a single spacecraft mission [Marklund et al., 2001].

[12] Recent observations at 4-7 $R_{E}$ geocentric distances by the four Cluster spacecraft [Marklund et al., 2001, 2004, 2007; Johansson et al., 2004, 2005, 2006; Karlsson et al., 2004; Figueiredo et al., 2005] shed new light in the temporal and spatial scale distribution of auroral fieldaligned potential structures. Event and statistical studies using Cluster data show that auroral electric field structures have perpendicular sizes (when mapped to the ionosphere) of a few kilometers to a few 10 s of kilometers with a distribution peak at $\sim 4-5 \mathrm{~km}$. Their lifetimes are at least $20 \mathrm{~s}$ and up to a few $100 \mathrm{~s}$. These results are a significant step ahead in our understanding of the physics of auroral arc formation and stability. However, the presence of a similar Cluster-like spacecraft formation at ionospheric altitudes would considerably contribute to our efforts in the separation of the spatial and temporal structure of auroral features. The recent Space Technology 5 (ST5) mission of three lowaltitude spacecraft in a string-of-pearls configuration has yielded some important observations in this direction [Spence et al., 2006], despite its short lifetime (March-June 2006).

[13] With the important physics of auroral arc structure and stability in mind, and with the inability of a single spacecraft to definitively delineate temporal and spatial variations, we appeal to the use of a unique data set provided by the dual DMSP F6 and F8 spacecraft. The purpose of this study is to examine the spatial and temporal characteristics of auroral particle precipitation features in order to identify the relationship between their latitudinal spatial scales and their temporal stability. In doing so, we follow the commonly accepted view that auroral precipitation features are the consequence of particle acceleration by parallel electric fields. However, a detailed comparison of measured precipitation and electric field scale sizes is beyond the scope of this study. The paper is organized as follows: section 2 gives an overview of the spacecraft and instrument characteristics, along with a description of the data set. Section 3 describes the mathematical techniques used to analyze the data. Section 4 contains the full analysis and results obtained from the application of the above techniques, and section 5 summarizes our conclusions and discusses some important aspects of auroral temporal and spatial structure.

\section{Data Set}

[14] A brief description of the spacecraft, the instruments and the physical quantities obtained from them, is given in this section. For a more detailed account of all the above see Hardy et al. [1984].

[15] The two DMSP satellites are in sun-synchronous, circular, polar orbits and are virtually co-orbital in the dawn- dusk plane; their cross-track separations rarely exceed $50 \mathrm{~km}$ at auroral latitudes (i.e., typically comparable to or less than the resistive scale length $\Lambda$ ). Owing to the diurnal rotation of the geomagnetic pole about the geographic pole, the two satellites have wide spatial coverage in geomagnetic coordinates. They both orbit at altitudes between 800 and $900 \mathrm{~km}$. A slight difference in their semimajor axes yields a fractional difference in their orbital periods; they differ by less than $40 \mathrm{~s}$ out of approximately $101 \mathrm{~min}$ periods. This leads to a racetrack effect in which the faster satellite (F6) "laps" the slower satellite (F8) regularly. The resonant interaction time is approximately $11 \mathrm{~d}$. Near closest approach, the separation gradually reduces to a minimum of about $10 \mathrm{~km}$, thereby offering for the first time simultaneous sampling of the low-altitude space particle environment at two nearly colocated positions. Measurements obtained as one satellite approaches the other along the same meridian, with in-track separations ranging from 10 to $1000 \mathrm{~km}$, are ideal for studying the spatial and temporal variability of auroral precipitation and form the basis of our analysis. The utility of this data set has already been shown by several case studies [Watermann et al., 1993; Jorgensen and Spence, 1997; Jorgensen et al., 1999; Boudouridis et al., 2001, 2002, 2003].

[16] The data we used in this study were obtained by the Geophysics Laboratory SSJ/4 instruments flown on both DMSP F6 and F8 spacecraft. These sensors have their look directions always oriented radially away from the Earth. They are identical in design, measuring the flux of precipitating electrons and ions in 20 energy channels, logarithmically spaced over the energy range of $30 \mathrm{eV}$ to $30 \mathrm{keV}$. They achieve this by using a set of four cylindrical curved plate electrostatic analyzers, arranged in two pairs. One pair counts electrons, in 10 channels between $30 \mathrm{eV}$ and $1000 \mathrm{eV}$ and 10 channels between $1 \mathrm{keV}$ and $30 \mathrm{keV}$, and the other pair counts ions in a similar way. The aperture size of the sensors allows for an angular response off the radial direction with full width at half maximum of $\Delta \alpha=1.7^{\circ}-$ $1.8^{\circ}$ perpendicular to the two cylindrical plates of the analyzer, and $\Delta \beta=5.4^{\circ}-6.8^{\circ}$ parallel to the cylindrical plates, for the low-energy channels. The respective values for the high-energy channels are $\Delta \alpha=3.4^{\circ}-4.3^{\circ}$ and $\Delta \beta=$ $4.7^{\circ}-5.2^{\circ}$. The incoming particles are therefore highly aligned along the radial direction. The detectors remain in each channel for a period of $98 \mathrm{~ms}$, with $2 \mathrm{~ms}$ left between steps to stabilize the voltage. The duty cycle of the SSJ/4 instrument yields a complete 20 point electron and ion spectrum once per second. This corresponds to a spatial resolution of approximately $7 \mathrm{~km}$ along the orbital track.

[17] Owing to the special operations required to retrieve concurrent data from the two spacecraft, only one of which is considered operational at a given time, the time span during which such data were made available was limited. This ultimately yielded a data set consisting of $11 \mathrm{~d}$ of useful conjunctions between the two satellites, spanning the period from September 1989 to April 1990. A total of 77 separate auroral zone crossings were analyzed for this study. The differential number fluxes for both electrons and ions from the two satellites for one of these crossings on 4 April 1990 are shown in Figure 1.

[18] For this study we concentrate on the analysis of the integral number and energy fluxes of electrons and ions. 


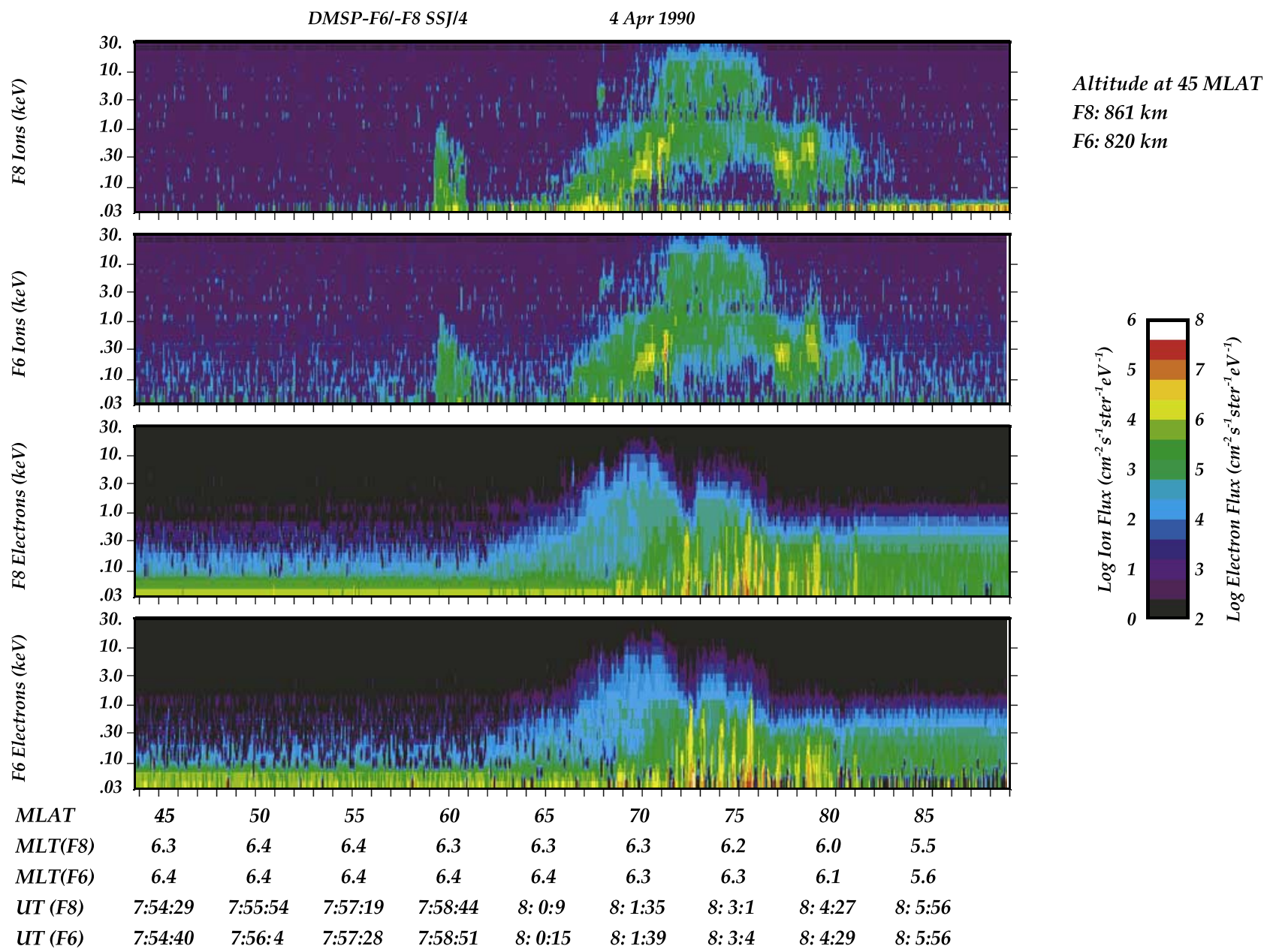

Figure 1. Differential number fluxes from the two DMSP spacecraft for $\sim 0754-0807$ UT on 4 April 1990. The time lag between the two satellites for this pass was $\sim 5 \mathrm{~s}$.

These quantities can be easily calculated, as a function of universal time (UT), from the differential number fluxes $j\left(E_{i}\right)$ of all energy channels, each with central energy $E_{i}$. The use of these quantities in single spacecraft statistical studies of electron and ion precipitation has been demonstrated by Hardy et al. [1985, 1989]. The calculated integral fluxes from each satellite were then transformed from data organized by constant time increments $\Delta t=1 \mathrm{~s}$, to data organized by constant magnetic latitude increments $\Delta l=$ $0.05^{\circ}$, for each auroral zone crossing, and plotted on the same magnetic latitude axis. The average time separation between the two spacecraft during each one of these crossings ranges from $1 \mathrm{~s}$ up to $\sim 2 \mathrm{~min}$. This format facilitates the identification of temporal variation, latitudinal motion, or persistence of discrete auroral features during each pass. Figure 2 shows the integral number flux plot for the same interval of Figure 1 on 4 April 1990.

\section{Mathematical Techniques}

[19] Visual examination of the combined DMSP F6 and F8 data sets, such as the example shown in Figure 2, indicates that auroral features of moderate to large spatial scales generally persist over timescales of a few tens of

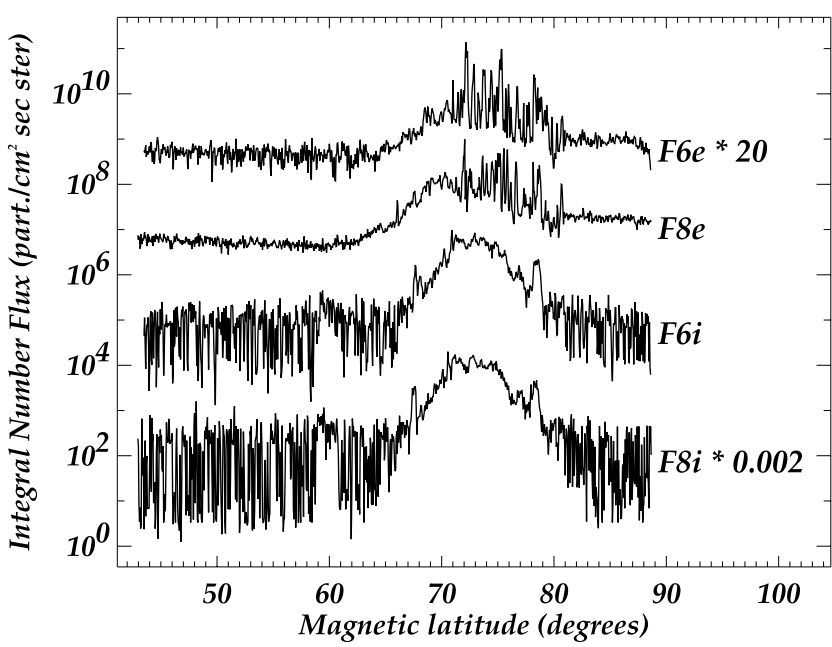

Figure 2. Integral number fluxes of electrons (top two curves) and ions (bottom two curves) from the two DMSP spacecraft for $\sim 0754-0807$ UT on 4 April 1990 as in Figure 1. The F6e and F8i curves have been shifted vertically by the factors shown for clarity. 


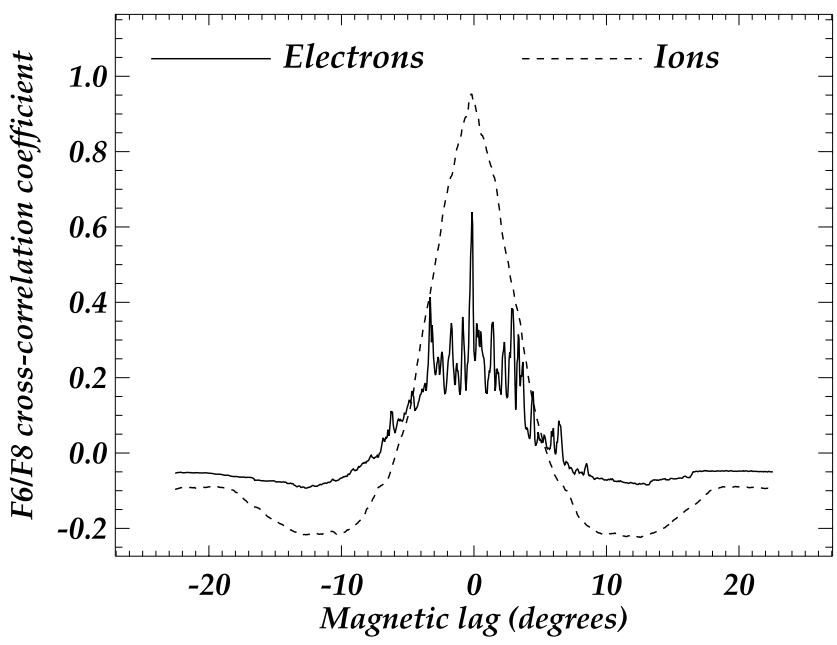

Figure 3. An example of a cross-correlation coefficient $\rho$. This is for the integral number fluxes of Figure 2, at $\sim 0754-0807$ UT on 4 April 1990. Notice the high variability and the relative smoothness of the electron and ion curves, respectively.

seconds. On the other hand, in many cases substantial variability is observed on timescales as short as a few seconds for smaller-scale features. To investigate the spatial structure and temporal stability of auroral features quantitatively, we employ two techniques of time series analysis appropriate for intercomparison of two independent time series, or in our case magnetic latitude series (MLS). These are cross-correlation analysis (CCA) and cross-spectral analysis (CSA). A more thorough description of these series analysis methods can be found in the work of Bendat and Piersol [1971, 1980] and Jenkins and Watts [1968].

\subsection{Cross-Correlation Analysis}

[20] This technique yields information on the correlation or "matching" of the precipitating electron or ion integral fluxes from the two satellites, as a function of the magnetic latitude shift $\lambda$ or lead distance between the two measurements. This is achieved by the introduction of a quantity called the cross-correlation coefficient (CCC), defined as

$$
\rho(\lambda)=\frac{C_{x y}(\lambda)}{\sqrt{C_{x x}(0) C_{y y}(0)}}, \quad-1 \leq \rho(\lambda) \leq 1
$$

The cross-covariance, $C_{x y}$ and autocovariance, $C_{x x}$ and $C_{y y}$, functions in equation (7) are given by

$$
\begin{aligned}
& C_{x y}(L)=\frac{1}{N} \sum_{k=0}^{N-L-1}\left(x_{k+L}-\bar{x}\right)\left(y_{k}-\bar{y}\right) \\
& C_{x x}(L)=\frac{1}{N} \sum_{k=0}^{N-L-1}\left(x_{k+L}-\bar{x}\right)\left(x_{k}-\bar{x}\right) \\
& C_{y y}(L)=\frac{1}{N} \sum_{k=0}^{N-L-1}\left(y_{k+L}-\bar{y}\right)\left(y_{k}-\bar{y}\right)
\end{aligned}
$$

where $L=\lambda \Delta l$ is the integer point shift and $N$ is the number of points in each MLS, $x_{k}(l)$ and $y_{k}(l)$, which represent any of the two integral fluxes, number or energy, for electrons or ions, measured by the two DMSP spacecraft. In the above $\bar{x}$ and $\bar{y}$ are the average values of these series.

[21] So essentially what the CCA does is shift the two series with respect to each other and then take the sum of the products of the respective series values at every point in the region of overlap between the two MLS. It then compares this cross-covariance function $C_{x y}(L)$, with the square roots of the unshifted autocovariance functions $C_{x x}(0)$ and $C_{y y}(0)$, as in equation (7), resulting in a value between -1 and 1 for the correlation coefficient. The more alike the two series are the higher positively this coefficient will be, maximizing at some magnetic latitude shift $\lambda_{\max }$ which reveals a motion of the entire precipitation structure during the time passed between the two measurements. This allows us to explore the variation of the cross-correlation between the two satellite measurements as a function of their average time separation or time lag which, as mentioned in section 2, varies between $1 \mathrm{~s}$ and about $2 \mathrm{~min}$. In the case where the two series have opposite sign values (after their average is subtracted) at most of the points in the overlap region, a negative value results for $\rho$, pointing to an anticorrelation of the two series at that magnetic latitude shift. In other words the CCC is a measure of how much agreement or disagreement exists between the two MLS, $x_{k}(l)$ and $y_{k}(l)$. An example of a CCC for electrons and ions for the integral number fluxes of Figure 2, at $\sim 0754$ 0807 UT on 4 April 1990, is shown in Figure 3.

\subsection{Cross-Spectral Analysis}

[22] While the CCA provides an important insight on the structure of auroral precipitation in terms of overall values, it does not do so as a function of frequency or spatial scale of the auroral features. At the same time it is not particularly helpful when it comes to exploring the temporal variability of auroral features. We will address the second question further in section 4 . In order to pursue the first problem we make use of a method called cross-spectral analysis which is a natural extension of the CCA. This second approach utilizes a spectral coherence technique which provides information on the temporal coherence or persistence of auroral features as a function of their latitudinal spatial dimension. To accomplish this, we use a quantity called coherence function $(\mathrm{CF})$ which is a function of frequency $f$, or inverse spatial scale, and is defined as

$$
\gamma_{x y}^{2}(f)=\frac{\left|G_{x y}(f)\right|^{2}}{G_{x x}(f) G_{y y}(f)}, \quad 0 \leq \gamma_{x y}^{2}(f) \leq 1
$$

The CF is a measure of how stable an auroral feature of a particular size is during the time passed between the two measurements.

[23] The cross-spectral density function, $G_{x y}$ and the autospectral density functions, $G_{x x}$ and $G_{y y}$, can be constructed as follows. The MLS, $x_{k}(l)$ and $y_{k}(l)$, are divided into $n_{d}$ records of equal length $T$. The Fourier transform of each one of these records is calculated, and the average of the appropriate for each case product of Fourier transforms, 
for every frequency, is computed over all records, as shown in equations (10)

$$
\begin{gathered}
G_{x y}(f)=\frac{2}{n_{d} T} \sum_{j=1}^{n_{d}} X_{j}^{*}(f, T) Y_{j}(f, T) \\
G_{x x}(f)=\frac{2}{n_{d} T} \sum_{j=1}^{n_{d}}\left|X_{j}(f, T)\right|^{2} \\
G_{y y}(f)=\frac{2}{n_{d} T} \sum_{j=1}^{n_{d}}\left|Y_{j}(f, T)\right|^{2}
\end{gathered}
$$

In these equations, $X_{j}(f, T)$ and $Y_{j}(f, T)$ are the Fourier transforms at frequency $f$ of the $j$ th record of length $T$ of the MLS $x_{k}(l)$ and $y_{k}(l)$, respectively. $X_{j}^{*}(f, T)$ in equation (10a) denotes the complex conjugate of $X_{j}(f, T)$.

[24] It is well known [e.g., Bendat and Piersol, 1980] that for a finite length record of data, in our case a MLS, the finite FT is given by

$$
X_{j}(f, T)=\int_{0}^{T} x_{j}(l) e^{-i 2 \pi f l} d l
$$

where $x_{j}(l)$ is the $j$ th record of our original MLS. The Fourier frequency space is sampled evenly at frequencies

$$
f=\frac{1}{T}, \frac{2}{T}, \frac{3}{T}, \ldots, \frac{1}{2 \Delta l}
$$

with $\Delta f=1 / T$ being the frequency resolution. The highest frequency sampled is the so-called Nyquist frequency, $f_{c}=$ $1 /(2 \Delta l)$, and it depends on our data resolution $\Delta l$, while the lowest frequency depends on the record length $T=n \Delta l$, $n$ being the number of points in the record. The inverse of the frequencies of equation (12) yields the latitudinal spatial scales of the auroral features we can study with this data set, ranging from $2 \Delta l=0.1^{\circ}$ to the record length $T$. The negative frequencies, although present in the FT computation of equation (11), were not included in our study since the spectral density functions of equations (10) are defined as "one-sided" spectral density functions, meaning that they are equal to 0 for $f<0$ and twice the corresponding "two-sided" spectral density functions for $f>0$.

[25] The CF has a function analogous to that of the CCC in terms of comparing the two series to find out how similar they are. The difference here is that the CF can do that as a function of frequency or inverse characteristic spatial scale of the auroral features involved, while the CCC gives the agreement or disagreement between the two series as a whole. In this scheme the spectral density functions have a role similar to the one of the covariance functions. The cross-spectral density function computes the average product of the number of features of a specific scale present in the two series in a similar way that the cross-covariance function calculates the average product of the two series' deviation from their mean values for their entire overlap range at a given magnetic latitude shift. This average product is then compared to its equivalents from the autospectral density functions through the CF. The more persistent the features of a particular scale are during the time interval elapsed from one measurement to the other, the closer to unity the $\mathrm{CF}$ for this scale will be. It becomes unity only when the two spacecraft see exactly the same number of the features in question during that pass. Examples of CF plots will be given in the next section where we will also show how to exploit an ensemble-averaged coherence technique to determine the temporal stability of features of certain spatial scale ranges.

\section{Analysis and Results}

[26] A certain amount of "cleaning" was performed on the data in order to obtain magnetic latitude series that are easy to use and at the same time contain the least amount of distorting artifacts. First, a minimum value for the integral number and energy fluxes was chosen for the times when there were zero counts in all energy channels. Instead of choosing an arbitrary value, we put the zero count threshold to the minimum nonzero value for that series. In this per series correction method we let the zero count measurements adjust to the precipitation level of the rest of the time series for that pass. We then splined the time series to a regularly spaced magnetic latitude grid, producing the MLS as mentioned in section 2. Another problem, especially with the F8 ion data, was the artificially elevated counts for the lowest energy channel which greatly affected the particle number fluxes. Since there was no nonarbitrary way to correct these erroneous measurements, we completely excluded the lowest energy channel from the integral flux calculations.

[27] We finally conducted one last cleaning operation by removing all the "spiky" data points from the MLS, attributing them to faulty or nonregular measurements. This was done manually for a few selected single-point data that had extremely high value compared to the adjacent data points. Their fluxes at the faulty energy channels were replaced by the average of the corresponding fluxes at the two neighboring points. This despiking is absolutely necessary for a correct evaluation of the correlation coefficients since an extremely high value appearing in only one of the two series can greatly alter the resulting correlation between the two MLS.

\subsection{Correlation Analysis}

[28] In this section we examine the individual and collective behavior of the cross-correlation coefficients. Emphasis is mainly given to the F6/F8 cross-correlation coefficient (or simply CCC), but some statements are made regarding the electron/ion cross-correlation coefficient (EICCC). This one is computed for each satellite in order to investigate the matching between the electron and ion components of the precipitation regime and its evolution with spacecraft separation.

[29] In Figure 3 we showed an example of a CCC for both electrons and ions. The electron curve exhibits a great deal of variability corresponding to the highly variable discrete electron features at auroral latitudes. As the two MLS shift with respect to each other, the CCC obtains a high value every time two or more high flux features are 


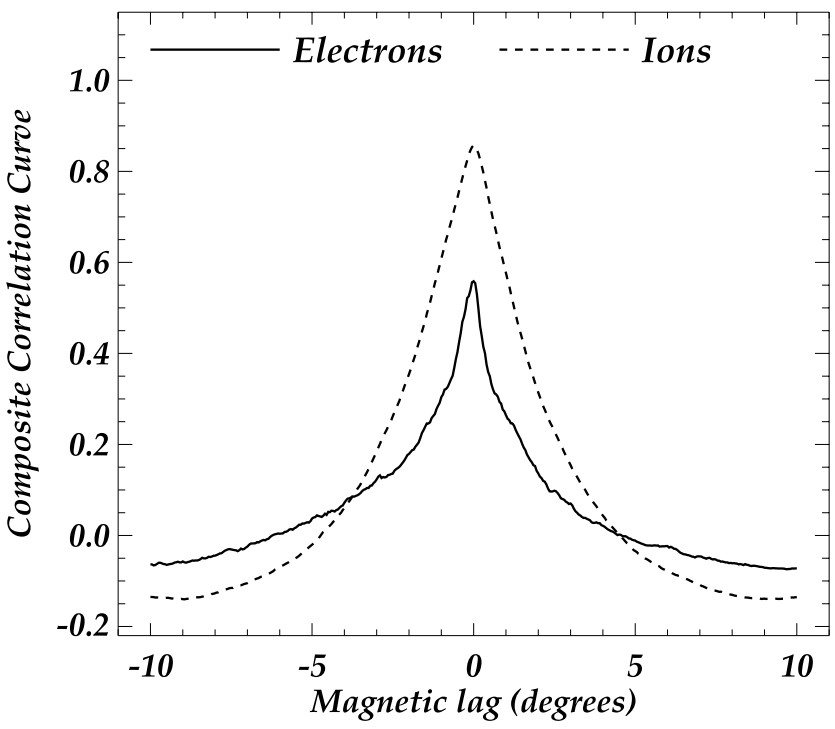

Figure 4. Average F6/F8 cross-correlation coefficients for the particle integral energy flux.

aligned. The ion curve on the other hand varies smoothly from its maximum value near zero magnetic latitude lag to the minimum negative values away from it. The lack of significant discrete features in the ion MLS is reflected in its CCC. The width of the various correlation peaks in Figure 3 is indicative of the average width of the respective MLS features. All the various scale sizes of these features contribute to the correlation peaks but it is the most common of the high flux ones that dominate the correlation curve. The electron correlation features of Figure 3 have widths of around $0.5^{\circ}-1^{\circ}$ of magnetic latitude or $50-100 \mathrm{~km}$ in spatial scales. The ion curve has a width of several degrees, and it corresponds to the width of the entire auroral zone due to the clear absence of discrete features of smaller latitudinal size. This characteristic scale is also evident in the electron curve as a broad background of slightly higher than zero CCC, on top of which the small-scale peaks reside.

[30] To gain better statistical understanding of the average scale sizes involved in the electron and ion precipitation regions, we averaged the correlation curves from all the DMSP passes. The results for electron and ion integral energy fluxes are shown in Figure 4. The electron curve width above the auroral zone background is again of the order of $1^{\circ}$ or $\sim 100 \mathrm{~km}$, consistent with the resistive scale length $\Lambda$ of equation (3), while the ion curve size is reflective of the typical auroral zone characteristics in the absence of discrete features.

[31] Let us now examine the statistical evolution of the CCC with varying time separation between the two spacecraft. Figure 5 shows $20 \mathrm{~s}$ block averages of the maximum of the integral energy flux CCC, calculated for each auroral pass, as a function of the satellite time lag. This time lag obviously varies during each pass, but an average for the entire auroral crossing is used for this plot. This is justified considering that for most auroral passes the time lag does not vary more than $\sim 5 \mathrm{~s}$ during the pass. Also, the correlation curve is dominated by the high-flux auroral zone features that occur right in the middle of the satellite pass where the smoothly varying time lag attains its average value. The time lag distribution in the $20 \mathrm{~s}$ blocks is as follows: 21 cases in the $0-20 \mathrm{~s}$ interval, 13 in the $>20-40 \mathrm{~s}$ interval, 14 in the $>40-60 \mathrm{~s}$ interval, 17 in the $>60-80 \mathrm{~s}$ interval, 7 in the $>80-100 \mathrm{~s}$ interval, and 5 in the $>100$ $120 \mathrm{~s}$ interval. Despite the smaller number of time lags in the two high time lag blocks, we can see that the electron correlation drops from about 0.8 at short time lags to less than 0.6 at higher time lag values, with a significant decrease around $80-100 \mathrm{~s}$. The ions seem to have a more or less constant value of around 0.9 , throughout the time lag range, accentuated by the high value in the $80-100 \mathrm{~s}$ block but still dropping slightly at high time lag values. At the same time, the ion correlation is always higher than the electron one.

[32] The observed differences of the average CCC for electrons and ions are in agreement with the different nature of the electron and ion auroral fluxes. It is expected that the correlation between two MLS will degrade with the passage of time, as the high-latitude precipitation environment responds to changes in the magnetosphere-ionosphere system and by extension to the interplanetary conditions. The timescales of auroral stability are different for electrons and ions due to the different dominant spatial scales of the features involved. This stability breaks down at around $90 \mathrm{~s}$ for the electron fluxes but seems to last longer for the ions, probably beyond the range of our spacecraft temporal separations. Also, the structured auroral zone electron precipitation is obviously much better correlated than the noisy low-latitude diffuse aurora region, but its $100 \mathrm{~km}$ size features which dominate the correlation curve are still highly variable compared with the slower varying ion precipitation. For this reason the ion CCC is higher than the electron one even for low time lag values, before the breakdown of the electron temporal stability.

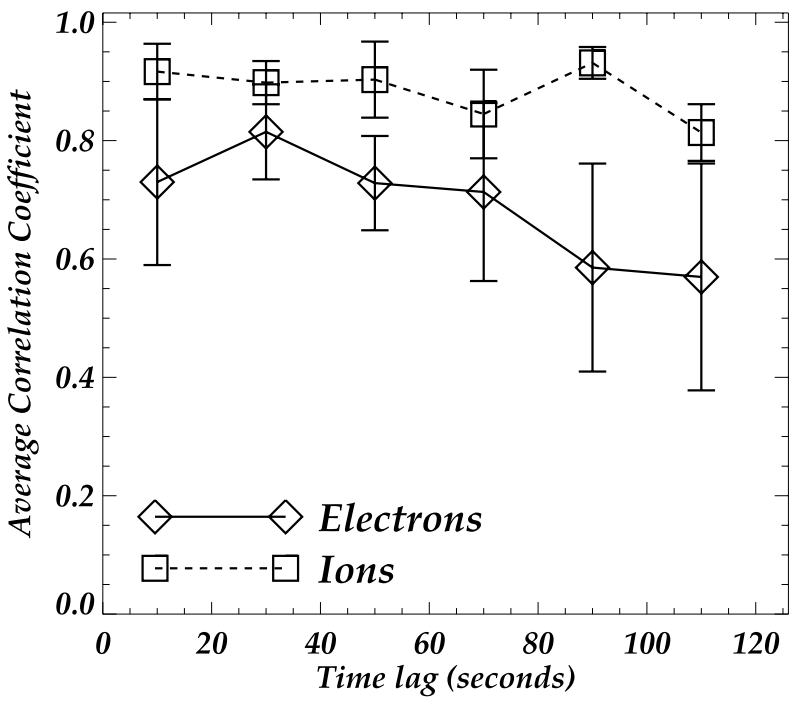

Figure 5. Cross-correlation block average as a function of spacecraft time separation for electron and ion integral energy fluxes. The error bars are standard deviations on the mean value. 


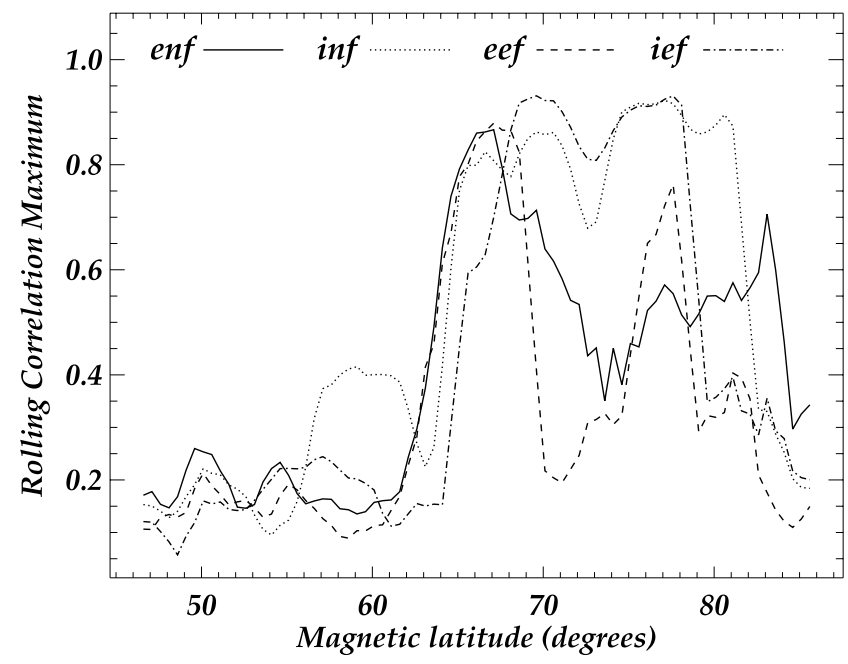

Figure 6. Rolling correlation coefficient for the satellite passes of Figure 2 (enf: electron number flux, inf: ion number flux, eef: electron energy flux, ief: ion energy flux). The "rolling" correlation window width is $6^{\circ}$ of magnetic latitude.

[33] The above statistical results refer to one CCC for the entire MLS of each satellite pass. One can also choose to concentrate only on the auroral zone part of the series where the most interesting high flux discrete structure exists, ignoring the uncorrelated low- and high-latitude diffuse precipitation. Application of the above principles to only that part, however, does not yield vastly different results due to the fact mentioned above that the CCC is dominated by the high energy flux part of the series. The low energy flux part simply does not contribute enough to the total for its absence to be a serious consequence.

[34] A far more interesting application of the correlation technique is the calculation of a "rolling" CCC. In this case a latitudinal window of a certain width slides or "rolls" through the MLS. At every point with latitude given by the center of the window the CCC is computed for the parts of the two series within this window, and its maximum is recorded as the CCC at this point. In this way a latitudinal variation of the CCC is obtained for every satellite pass, and a clear distinction of the correlated and uncorrelated parts can be made.

[35] An example of the rolling CCC with latitudinal window width of $6^{\circ}$ is shown in Figure 6 . The solid and dotted curves correspond to the electron and ion number flux rolling correlations, respectively, while the dashed and dot-dashed curves show the respective energy flux rolling correlations. It can be seen that all correlations have low values at low latitudes, which sharply increase as soon as the rolling window starts including higher auroral zone fluxes. The significant difference between the electron and ion curves is that the electron CCC drops to medium values when the discrete features are included in the calculation, while the ion CCC remains high throughout the auroral zone crossing. This observation clearly explains the different average values for electrons and ions shown in Figure 5. Since the CCC for the entire MLS is dominated by the higher fluxes, and these occur in the discrete part of the series for the electrons, it is those medium range correlations that appear in an average CCC plot. The part immediately equatorward of the discrete structure correlates better but is suppressed in the average. The ions on the other hand do not suffer from such variable structure, maintaining a high correlation all along. At the end of the auroral zone both particles return to low CCC values due to the uncorrelated polar rain.

[36] We can now explore this spatial variation of the rolling $\mathrm{CCC}$ together with its temporal variation described by the time separation of the two spacecraft. Figure 7 depicts the electron and ion integral energy flux rolling CCC (also with latitudinal window width of $6^{\circ}$ ) for all available passes. Each trace on these images is a single pass with the CCC color-coded as a function of magnetic latitude and time lag. Notice that the smaller latitudinal window used for the rolling CCC computation allows for a finer determination of the time lag between the spacecraft rather than the one average value for each pass used before. Two points that mentioned before are also confirmed here. First, on the average the electron correlations fall with increasing time lag, while the ion ones remain more or less constant. Second, the highest electron correlations occur below $\sim 70^{\circ}$ magnetic latitude, in the unstructured part of the auroral zone. In contrast, the ions exhibit a wide range of stability from $60^{\circ}$ all the way to $\sim 80^{\circ}$.

[37] Finally, the EICCC is calculated for every pass and for both spacecraft. This has generally a lower magnitude, around $0.4-0.7$, more or less unchanged over our time lag range. The average correlation curve has a width of about $6^{\circ}$, significantly wider than the average CCC of Figure 4. Both these features are expected considering the fact that the electron and ion data correlate best when their respective auroral zones align with each other. The average correlation curve width is representative of the auroral zone width rather than individual features within it. However, it is the very presence of these discrete, variable features in only one of the two MLS, namely the electrons, that brings the magnitude of the EICCC down.

\subsection{Spectral Analysis}

[38] As mentioned in section 3.2, the CCC determines the stability of particle precipitation in terms of overall values for the entire MLS or parts of it but not separately for features of different spatial scales. The latter is achieved with the calculation of the coherence function. For this purpose we divided each MLS into several records, each with $n=180$ points or length $T=n \Delta l=9^{\circ} \simeq 1000 \mathrm{~km}$. This is the largest latitudinal scale we can sample. The smallest one is given by $2 \Delta l=0.1^{\circ} \simeq 11 \mathrm{~km}$, with all the other spatial scales in between according to the inverse of equation (12). To examine the temporal variation of the $\mathrm{CF}$, we arrange the individual auroral crossings in groups according to the average time lag between the two satellites during each crossing. We use four time lag ranges of $30 \mathrm{~s}$ length each, up to the maximum available of about $2 \mathrm{~min}$. We then calculate the $\mathrm{CF}$ for each pass and average the results for all the passes within each $30 \mathrm{~s}$ time lag interval.

[39] Figure 8 shows the resulting average CF for electron and ion integral energy fluxes as a function of characteristic spatial scale of the auroral features. The CF for all time lag 

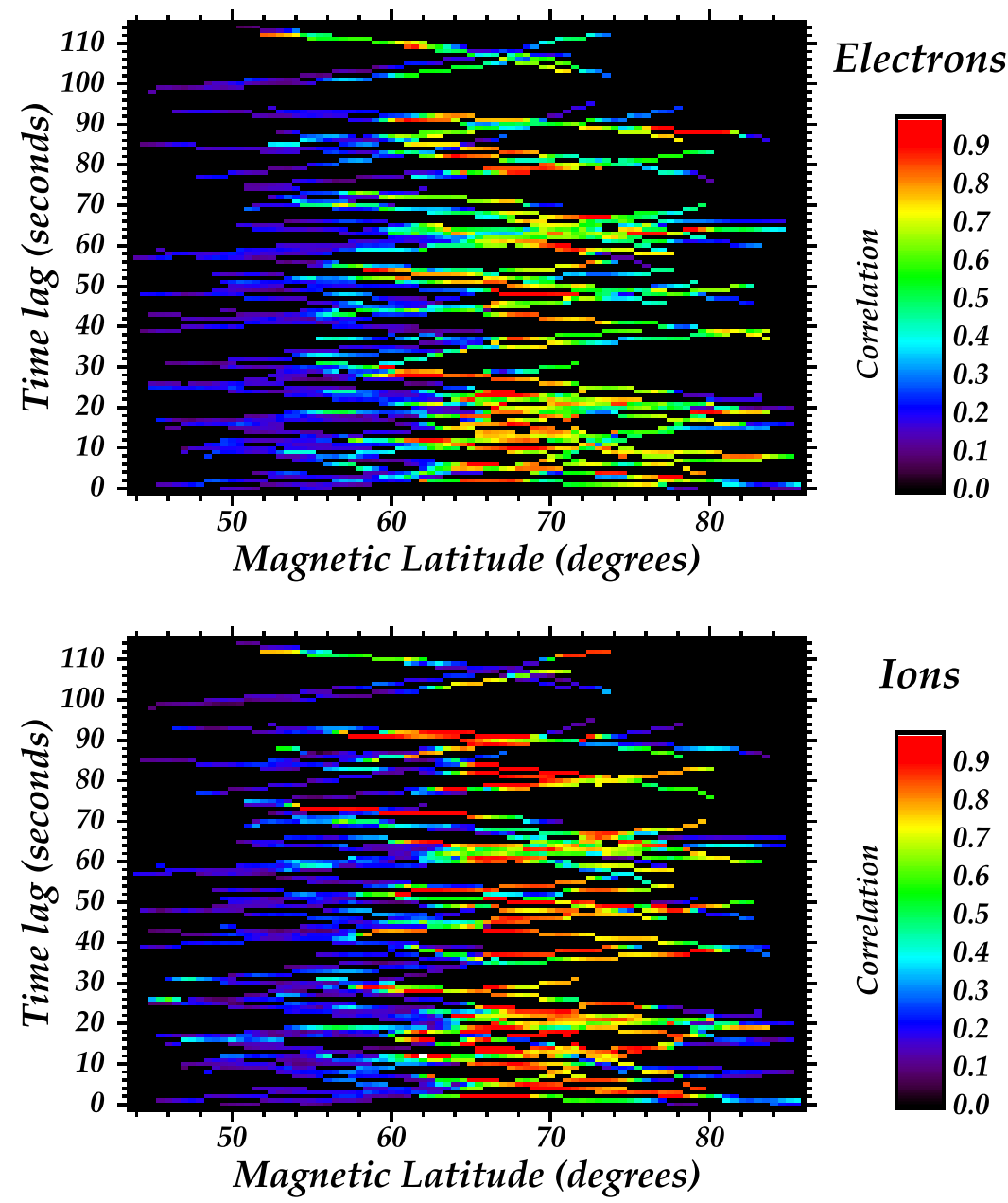

Figure 7. Temporal and spatial variation of the rolling CCC for the integral energy fluxes. The rolling correlation window width is $6^{\circ}$ of magnetic latitude.

ranges falls from an initial high value at large spatial scales $(>100 \mathrm{~km})$, to a lower and more variable value near the smaller scales $(<50 \mathrm{~km})$. The transition occurs between 50 and $100 \mathrm{~km}$, consistent again with the resistive scale length $\Lambda$. The drop is more apparent for the ion data, $\Delta \gamma \simeq 0.4-$ 0.5 , but is also present on the electron curves, $\Delta \gamma \simeq 0.2-$ 0.3 . This again can be due to the obvious scarcity of smaller features in the ion fluxes, in contrast with the electron ones.

[40] The temporal evolution of the CF curves has an interesting behavior. The electron coherence first falls for all frequencies in the 30-60 s time lag range but then bounces up to its original high value in the $60-90 \mathrm{~s}$ range. This is also the case, to a less extent though, for the low-frequency ion curves. This phenomenon could be attributed to periodicities in the stability of the auroral features consistent with the $\sim 1$ min timescales mentioned in the introduction, more so for the variable electron data than the ion data.

[41] A more important observation is the clear drop of the $\mathrm{CF}$ in the last 90-120 s range. This indicates the break down of auroral stability at around $90 \mathrm{~s}$ as pointed out in section 4.1. The one thing to notice about this drop is that it is scale-dependent for the ions but not the electrons. The electron $\mathrm{CF}$ diminishes for all frequencies examined during this last time lag range. The ion $\mathrm{CF}$, on the other hand, is already low for every time lag range in the high-frequency end of the spectrum, but the destabilization of the lowfrequency part $(>100 \mathrm{~km})$ occurs only after about $90 \mathrm{~s}$ spacecraft time separation.

\section{Summary and Discussion}

[42] In this work we investigated the spatial and temporal scales of high-latitude particle precipitation. The identification of these scales is of great importance in understanding the physical processes that contribute to the dynamics of the auroral ionosphere. We made use of a unique data set of particle fluxes from two co-orbital DMSP satellites with varying in-track separation. This enables us to systematically examine the temporal evolution of the auroral stability of features of various sizes. To achieve our goals, we employed two time series analysis techniques, correlation analysis and spectral analysis. We can summarize our conclusions as follows:

[43] 1. The correlation analysis shows that the electron precipitation in the auroral zone consists mainly of $100 \mathrm{~km}$ features that are quite variable in nature. They reside on a broader lower-frequency background. The ion fluxes have no such small-scale component but are dominated by a less 

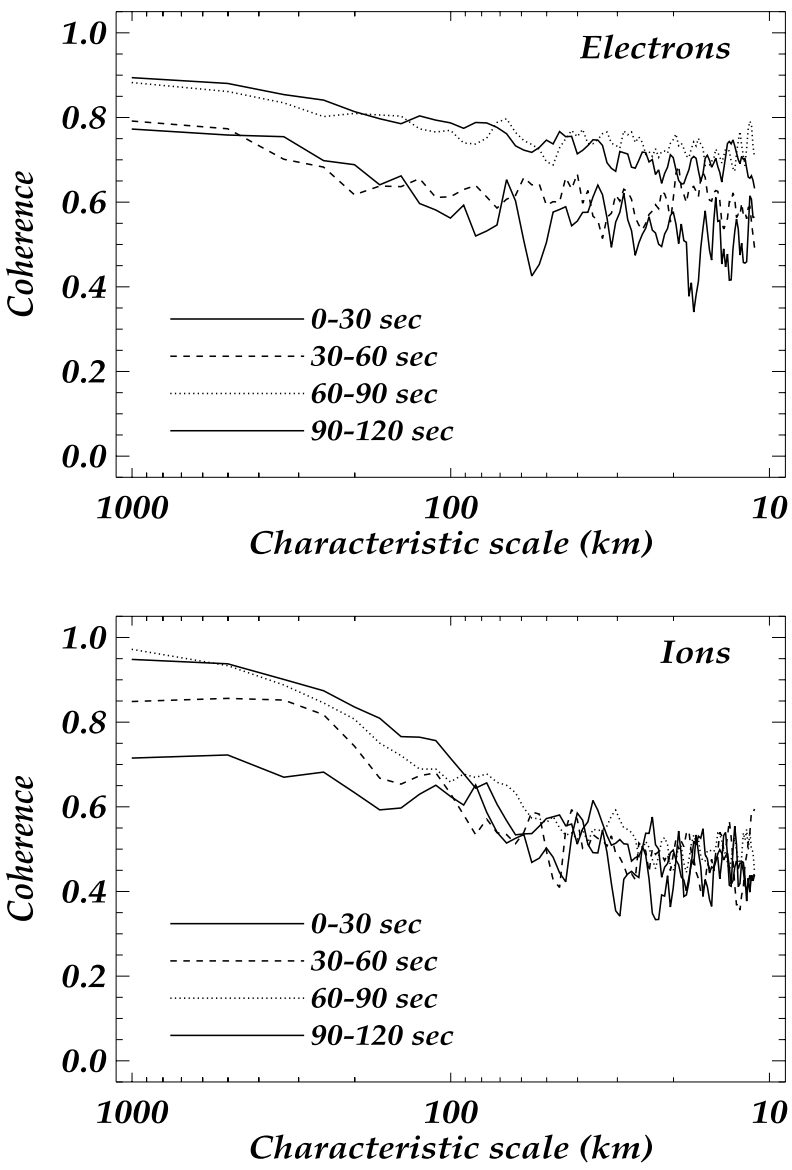

Figure 8. Coherence function for the particle integral energy fluxes.

variable auroral-zone-wide precipitation of a few degrees in latitudinal width.

[44] 2. The average electron CCC declines at around $90 \mathrm{~s}$ of spacecraft separation, signifying a breakup of the auroral stability at this timescale, while the ions show signs of a smaller decline around the same time lag. The ion CCC is higher than that of the electrons at all time lags.

[45] 3. The application of a "rolling" correlation shows a highly correlated ion precipitation throughout the auroral zone, with one virtually uncorrelated at lower latitudes. The electron correlation reaches its peak at the equatorward part of the auroral zone, reducing to medium range values over the more poleward high-flux features.

[46] 4. The coherence analysis shows that the CF (which is a measure of stability) drops from its original high values at high spatial scales to lower values for smaller scales, with the break occurring at around $50-100 \mathrm{~km}$, for all time lag ranges. This drop is more significant for the ion than the electron data.

[47] 5. The temporal evolution of the $\mathrm{CF}$ is consistent with the above mentioned stability breakup at $90 \mathrm{~s}$, for both electrons and ions. However, it seems to be scale-dependent for the ion features but scale-independent for the electron features. It also shows signs of periodic behavior with a period of $1 \mathrm{~min}$, but this phenomenon requires more thorough investigation.
[48] As mentioned earlier, the characteristic spatial scale for stable auroral features suggested by our analysis agrees well with the scale length obtained by the adiabatic kinetic theory of auroral formation, $\Lambda \sim 50-100 \mathrm{~km}$ [Chiu and Cornwall, 1980; Lyons, 1980]. However, many theoretical models of auroral arc thickness predict scales smaller than

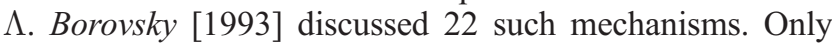
three of them generate features greater than $50 \mathrm{~km}$, namely shear in the low-latitude boundary layer $(130 \mathrm{~km})$, shear in the central plasma sheet $(51 \mathrm{~km})$, and electrostatic fluid turbulence in the central plasma sheet $(120 \mathrm{~km})$. Our results suggest that features produced by these processes are more stable than the smaller features. In other words, the imperfect mapping of the magnetospheric electric field to the ionosphere for features less than $50 \mathrm{~km}$ [Weimer et al., 1985] seems to be an adverse factor to their stability regardless of the way they are generated.

[49] Let us now turn our attention to the timescales (4)(6) discussed by Cornwall [1990]. Of these only $T_{E \times B}$ is explicitly dependent on the latitudinal spatial dimension of an auroral feature. We also noted above that the temporal variation of the ion $\mathrm{CF}$ is scale-dependent but the electron one is not. We can give the following interpretation to our results. For small ion features, equation (6) gives drift times shorter than the other two timescales and therefore brings the $\mathrm{CF}$ down to low values almost immediately. As the scale size increases, the drift time grows and somewhere above $100 \mathrm{~km}$ it becomes larger than the smallest of the other two timescales. Hence at higher scales the ion auroral features become unstable due to either the ionization or the dissociative recombination mechanism with a size-independent timescale of $\sim 90 \mathrm{~s}$ as shown in Figure 8. In the case of electrons, $\mathbf{E} \times \mathbf{B}$ drift transport seems to be ineffective in immediately reducing the $\mathrm{CF}$ for small features. As observed by Weimer et al. [1985] and theoretically confirmed by Chiu [1986], smaller electron features are associated with smaller potential drops. According to equation (6), this will increase the drift transport time, probably above the constant $T_{I}$ or $T_{D R}$ for all spatial scales, causing the CF to evolve in a "uniform" way. The suggested behavior of all the relevant timescales as a function of spatial scales is schematically illustrated in Figure 9.

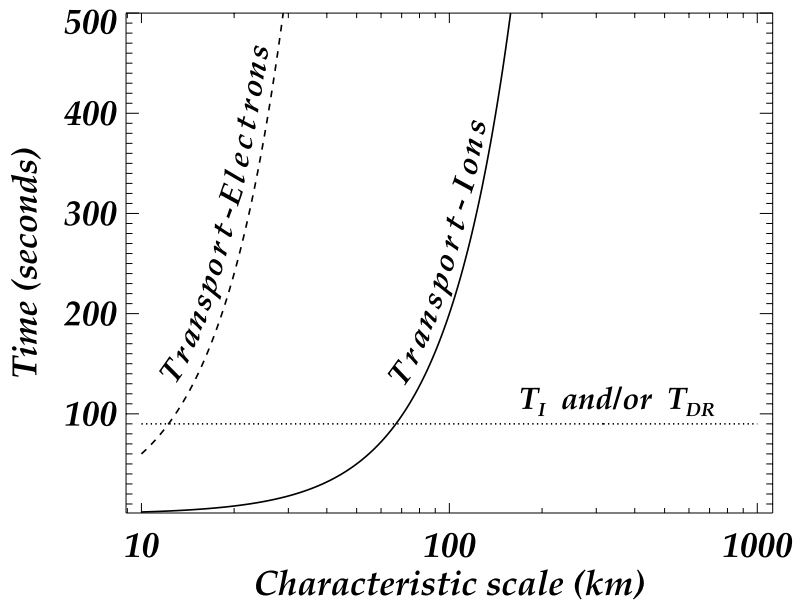

Figure 9. Suggested behavior of ionospheric timescales as a function of characteristic spatial scale. 
[50] On the basis of our CF results we can therefore claim that drift transport plays an important role in ion auroral stability, whereas the electron stability is dominated by the other two timescales. Yet again our data set is limited to time lags less than $2 \mathrm{~min}$, pretty close to the coherence dropoff point, with few passes in the last time lag range. A larger data set would sufficiently resolve any ambiguities about the temporal evolution of auroral stability by extending both the number of auroral crossings and spacecraft time separation.

[51] Another problem we have to acknowledge is that the smallest spatial scale we can probe with the DMSP measurements is around $10 \mathrm{~km}$. Most of the mechanisms outlined by Borovsky [1993] yield scale sizes below this limit. A separate study including only these small-size features should be conducted. This will require at least two spacecraft with faster instrument cycle, like FAST, to improve the spatial resolution. The ideal solution would be a small fleet of colocated, low-altitude, FAST-type spacecraft with slightly different periods. This would provide an excellent data set for the study of the stability of the smaller auroral features, using the two methods we employed in this work.

[52] Despite its shortcomings, low spatial resolution, limited time lag range, and low statistics, the unique data set used in this work demonstrates overwhelmingly the utility of the two-point measurements in the effort to separate the temporal and spatial structure of auroral particle precipitation. The multitude of one-point measurements will always suffer from temporal/spatial ambiguities, but multiple-point measurements can readily address these problems, not only in the high-latitude precipitation environment but throughout the magnetosphere-ionosphere system.

[53] Acknowledgments. The authors wish to thank David J. Gorney of the Aerospace Corporation for many useful discussions. This work was supported by NASA grant NNG05GK65G.

[54] Amitava Bhattacharjee thanks Sónia Liléo and another reviewer for their assistance in evaluating this paper.

\section{References}

Basu, Su., S. Basu, E. MacKenzie, P. F. Fougere, W. R. Coley, N. C. Maynard, J. D. Winningham, M. Sugiura, W. G. Hanson, and W. R. Hoegy (1988), Simultaneous density and electric field fluctuation spectra associated with velocity shears in the auroral regions of the ionosphere, J. Geophys. Res., 93, 115-136.

Bendat, J. S., and A. G. Piersol (1971), Random Data: Analysis and Measurement Procedures, Wiley-Interscience, New York.

Bendat, J. S., and A. G. Piersol (1980), Engineering Applications of Correlation and Spectral Analysis, Wiley-Interscience, New York.

Boehm, M. H., G. Paschmann, J. Clemmons, G. Haerendel, L. Eliasson, and R. Lundin (1994), Freja observations of narrow inverted-V electron precipitation by the two-dimensional electron spectrometer, Geophys. Res. Lett., 21, 1895-1898.

Borovsky, J. (1993), Auroral arc thicknesses as predicted by various theories, J. Geophys. Res., 98, 6101-6138.

Boudouridis, A., H. E. Spence, and T. G. Onsager (2001), Investigation of magnetopause reconnection models using two colocated, low-altitude satellites: A unifying reconnection geometry, J. Geophys. Res., 106, 29,451-29,466.

Boudouridis, A., H. E. Spence, and T. G. Onsager (2002), A new look at the pulsed reconnection model of the dayside magnetopause, Adv. Space Res., 30(10), 2295-2300.

Boudouridis, A., H. E. Spence, and T. G. Onsager (2003), Formation of the LLBL in the context of a unifying magnetopause reconnection mechanism, in Earth's Low-Latitude Boundary Layer, Geophys. Monogr. Ser, vol. 133, edited by P. T. Newell and T. G. Onsager, pp. 131-138, AGU, Washington, D. C.

Chiu, Y. T. (1986), A simple kinetic theory of auroral arc scales, J. Geophys. Res., 91, 204-210.
Chiu, Y. T. (1987), Resonant Alfvén waves on auroral field lines, J. Geophys. Res., 92, 3402-3408.

Chiu, Y. T., and J. M. Cornwall (1980), Electrostatic model of a quiet auroral arc, J. Geophys. Res., 85, 543-556.

Chiu, Y. T., and M. Schulz (1978), Self-consistent particle and parallel electric field distributions in the magnetospheric-ionospheric auroral region, J. Geophys. Res., 83, 629-642.

Chiu, Y. T., A. L. Newman, and J. M. Cornwall (1981), On the structures and mapping of auroral electrostatic potentials, J. Geophys. Res., 86, $10,029-10,037$

Cornwall, J. M. (1988), Exact solutions and low-frequency instability of the adiabatic auroral arc model, J. Geophys. Res., 93, 11,429-11,435.

Cornwall, J. M. (1990), Low-frequency ionization-driven instability of a discrete auroral arc model, J. Geophys. Res., 95, 19,079-19,088.

Ergun, R. E., et al. (1998), FAST satellite observations of electric field structures in the auroral zone, Geophys. Res. Lett., 25, 2025-2028.

Ergun, R. E., L. Anderson, D. S. Main, Y.-J. Su, C. W. Carlson, J. P. McFadden, and F. S. Mozer (2002), Parallel electric fields in the upward current region of the aurora: Indirect and direct observations, Phys. Plasmas, 9, 3685-3694.

Ergun, R. E., L. Anderson, D. S. Main, Y.-J. Su, D. L. Newman, M. V. Goldman, C. W. Carlson, A. J. Hull, J. P. McFadden, and F. S. Mozer (2004), Auroral particle acceleration by strong double layers: The upward current region, J. Geophys. Res., 109, A12220, doi:10.1029/ 2004JA010545.

Figueiredo, S., G. T. Marklund, T. Karlsson, T. Johansson, Y. Ebihara, M. Ejiri, N. Ivchenko, P.-A. Lindqvist, H. Nilsson, and A. Fazakerley (2005), Temporal and spatial evolution of discrete auroral arcs as seen by Cluster, Ann. Geophys., 23, 2531-2557.

Gorney, D. J. (1989), A study of the spatial scales of discrete auroral arcs, in Physics of Space Plasmas, edited by T. Chang, pp. 385-390, Scientific, Cambridge, U. K

Hardy, D. A., L. K. Schmitt, M. S. Gussenhoven, F. J. Marshall, H. C. Yeh, T. L. Schumaker, A. Huber, and J. Pantazis (1984), Precipitating electron and ion detectors (SSJ/4) for the block 5D/flight 6-10 DMSP satellites: Calibration and data presentation, Rep. AFGL-TR-84-0314, Air Force Geophys. Lab., Hanscom Air Force Base, Mass.

Hardy, D. A., M. S. Gussenhoven, and E. Holeman (1985), A statistical model of auroral electron precipitation, J. Geophys. Res., 90, 4229-4248. Hardy, D. A., M. S. Gussenhoven, and D. Brautigam (1989), A statistical model of auroral ion precipitation, J. Geophys. Res., 94, 370-392.

Jenkins, G. M., and D. G. Watts (1968), Spectral Analysis, Holden-Day, San Fransisco, Calif.

Johansson, T., S. Figueiredo, T. Karlsson, G. Marklund, A. Fazakerley, S. Buchert, P.-A. Lindqvist, and H. Nilsson (2004), Intense high-altitude auroral electric fields-Temporal and spatial characteristics, Ann. Geophys., 22, 2485-2495.

Johansson, T., T. Karlsson, G. Marklund, S. Figueiredo, P.-A. Lindqvist, and S. Buchert (2005), A statistical study of intense electric fields at 4-7 $R_{E}$ geocentric distance using Cluster, Ann. Geophys., 23, 2579-2588.

Johansson, T., G. Marklund, T. Karlsson, S. Liléo, P.-A. Lindqvist, A. Marchaudon, H. Nilsson, and A. Fazakerley (2006), On the profile of intense high-altitude auroral electric fields at magnetospheric boundaries, Ann. Geophys., 24, 1713-1723.

Jorgensen, A. M., and H. E. Spence (1997), On separating space and time variations of auroral precipitation: Dual DMSP-F6 and -F8 observations, Adv. Space Res., 20(3), 453-456.

Jorgensen, A. M., H. E. Spence, T. J. Hughes, and D. McDiarmid (1999), A study of Omega bands and PS- 6 pulsations on the ground, at low altitude and at geostationary orbit, J. Geophys. Res., 104, 14,705-14,715.

Karlsson, T., and G. T. Marklund (1996), A statistical study of intense lowaltitude electric fields observed by Freja, Geophys. Res. Lett., 23, $1005-$ 1008 .

Karlsson, T., G. T. Marklund, S. Figueiredo, T. Johansson, and S. Buchert (2004), Separating spatial and temporal variations in auroral electric and magnetic fields by Cluster multipoint measurements, Ann. Geophys., 22, $2463-2472$.

Knight, S. (1973), Parallel electric fields, Planet. Space Sci., 21, 741-750.

Knudsen, D. J., E. F. Donovan, L. L. Cogger, B. Jackel, and W. D. Shaw (2001), Width and structure of mesoscale optical auroral arcs, Geophys. Res. Lett., 28, 705-708.

Lotko, W., B. U. O. Sonnerup, and R. L. Lysak (1987), Nonsteady boundary layer flow including ionospheric drag and parallel electric fields, J. Geophys. Res., 92, 8635-8648.

Lyons, L. R. (1980), Generation of large-scale regions of auroral currents, electric potentials, and precipitation by the divergence of the convection electric field, J. Geophys. Res., 85, 17-24.

Lyons, L. R. (1981), Discrete aurora as the direct result of an inferred highaltitude generating potential distribution, J. Geophys. Res., 86, 1-8. 
Lyons, L. R. (1992), Formation of auroral arcs via magnetosphere-ionosphere coupling, Rev. Geophys., 30, 93-112.

Lysak, R. L. (1990), Electrodynamic coupling of the magnetosphere and ionosphere, Space Sci. Rev., 52, 33-87.

Maggs, J. E., and T. N. Davis (1968), Measurements of the thicknesses of auroral structures, Planet. Space Sci., 16, 205-209.

Marklund, G. T., et al. (2001), Temporal evolution of the electric field accelerating electrons away from the auroral ionosphere, Nature, 414, $724-727$.

Marklund, G. T., T. Karlsson, S. Figueiredo, T. Johansson, P. A. Lindqvist, M. André, S. Buchert, L. M. Kistler, and A. Fazakerley (2004), Characteristics of quasi-static potential structures observed in the auroral return current region by Cluster, Nonlinear Proc. Geophys., 11, 709-720.

Marklund, G. T., T. Johansson, S. Lileo, and T. Karlsson (2007), Cluster observations of an auroral potential and associated field-aligned curren reconfiguration during thinning of the plasma sheet boundary layer, J. Geophys. Res., 112, A01208, doi:10.1029/2006JA011804.

Newell, P. T., K. M. Lyons, and C.-I. Meng (1996), A large survey of electron acceleration events, J. Geophys. Res., 101, 2599-2614.

Reiff, P., H. Collin, J. Craven, J. Burch, J. Winningham, E. Shelley, L. Frank, and M. Friedman (1988), Determination of auroral electrostatic potentials using high- and low-altitude particle distributions, J. Geophys. Res., 93 , $7441-7465$
Spence, H. E., R. J. Strangeway, G. Le, and J. D. Slavin (2006), In situ estimates of auroral current sheet structure and dynamics using threepoint ST5 magnetometer observations: A status report, Eos Trans. $A G U, 87(52)$, Fall Meet. Suppl., Abstract SM11A-0301.

Vedin, J., and K. Rönnmark (2005), Electrostatic potentials in the downward auroral current region, J. Geophys. Res., 110, A08207, doi:10.1029/ 2005JA011083.

Watermann, J., O. De la Beaujardiere, and H. E. Spence (1993), Space-time structure of the morning aurora inferred from coincident DMSP-F6, -F8 and Sondrestrom incoherent scatter radar observations, J. Atmos. Terr. Phys., 55, 1729-1739.

Weimer, D. R., C. K. Goertz, D. A. Gurnett, N. C. Maynard, and J. L. Burch (1985), Auroral zone electric fields from DE 1 and 2 at magnetic conjunctions, J. Geophys. Res., 90, 7479-7494.

Weimer, D. R., D. A. Gurnett, C. K. Goertz, J. D. Menietti, J. L. Burch, and M. Sugiura (1987), The current-voltage relationship in auroral current sheets, J. Geophys. Res., 92, 187-194.

A. Boudouridis, Department of Atmospheric and Oceanic Sciences, University of California, Los Angeles, 405 Hilgard Avenue, Los Angeles, CA 90095, USA. (thanasis@atmos.ucla.edu)

H. E. Spence, Center for Space Physics, Boston University, 725 Commonwealth Avenue, Boston, MA 02215, USA. (spence@bu.edu) 